\title{
Initial conditions of formation of starburst clusters: constraints from stellar dynamics
}

\author{
Sambaran Banerjee ${ }^{1,2}$ \\ ${ }^{1}$ Argelander-Institut für Astronomie \\ Auf dem Hügel 71, D-53121 Bonn, Germany \\ email: sambaran@astro.uni-bonn.de \\ ${ }^{2}$ Helmholtz-Instituts für Strahlen- und Kernphysik \\ Nussallee 14-16, D-53115 Bonn, Germany
}

\begin{abstract}
How starburst clusters form out of molecular clouds is still an open question. In this article, I highlight some of the key constraints in this regard, that one can get from the dynamical evolutionary properties of dense stellar systems. I particularly focus on secular expansion of massive star clusters and hierarchical merging of sub-clusters, and discuss their implications vis-á-vis the observed properties of young massive clusters. The analysis suggests that residual gas expulsion is necessary for shaping these clusters as we see them today, irrespective of their monolithic or hierarchical mode of formation.
\end{abstract}

Keywords. galaxies: star clusters, stellar dynamics, stars: formation, methods: n-body simulations, methods: numerical, ISM: clouds

\section{Introduction: birth conditions of young massive clusters}

Star clusters are found in our Milky Way and in all external galaxies with increasing detail. However, how they form in the first place is still one of the most important challenges of the cosmos. A key question of wide debate is how the exposed clusters' parsec-scale, centrally-pronounced, near-spherical shape, observed at all ages $\gtrsim 1 \mathrm{Myr}$, can be explained. This is in direct contrast with the irregular and much more extended (10s of parsecs) structure of molecular clouds, where stars form via gravitational fragmentation of dense substructures. An apparent lack of an age spread among the members of the youngest star clusters (see, e.g., Bastian \& Silva-Villa 2013) indicates that these stars form in a "burst" over a short period of time. This, in turn, implies that short-timescale dynamical processes, e.g., violent relaxation (Spitzer 1987), immediately or simultaneously follow the formation of the proto-stars, which shape the newly born cluster.

The unprecedented spatial and spectral resolution in IR and sub-mm wavelengths with Herschel and ALMA has revealed intricate filamentary networks inside dense regions of molecular clouds (see André et al. 2014 for a review). Such observations generally infer that the (projected) widths of the individual filaments and their junctions are as compact as $0.1-0.3$ pc. Both theoretical and observational studies suggest that groups of protostars preferentially form within these filaments and at their junctions (Schneider et al. 2012; André et al. 2014).

In Banerjee \& Kroupa (2015a), the role of violent relaxation in shaping a young cluster is studied. It is demonstrated that in order to have a single cluster in dynamical equilibrium right from Myr-age, as for massive starburst clusters, the stellar system involved in the mass assembly process must be "near-monolithic". This implies the formation of 


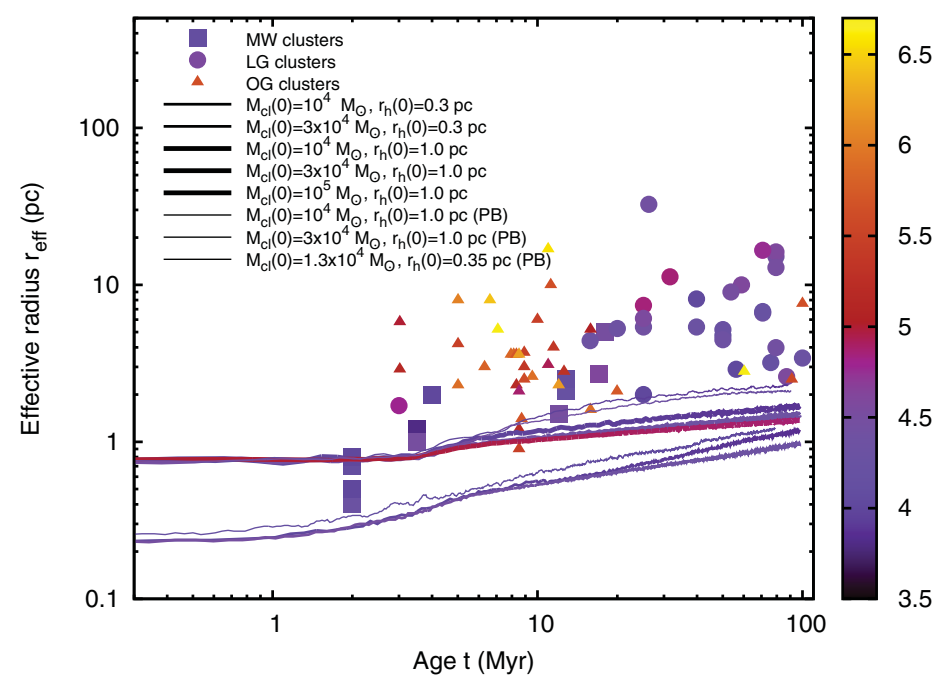

Figure 1. Effective radius, $r_{\text {eff }}$, vs. age, $t$, plot (data from Portegies Zwart et al. 2010) for observed young massive clusters (YMCs) in the Milky Way (MW), the Local Group (LG) and external galaxies $(\mathrm{OG})$ which are distinguished by different filled symbols. The symbols are colour-coded according to the clusters' respective photometric mass, $\log _{10}\left(M_{\text {phot }} / M_{\odot}\right)$. Overlaid are the computed curves for the evolution of projected half-mass radius (or effective radius), $r_{\text {eff }}(t)$, for model star clusters, without residual gas expulsion ('PB' $\Rightarrow$ primordial binaries included), which are also colour-coded by their instantaneous bound mass.

either a single (monolithic) proto-cluster within a dense molecular clump or of several sub-clusters that merge in $\lesssim 1$ Myr from parsec-scale separation.

In this study, useful constraints are obtained for assembling massive clusters given their birth conditions, as summarized above, and the physics of dynamical evolution. Especially, I shall shed light on one of the most widely debated question regarding massive cluster formation, namely, the relevance of primordial gas blow-out by stellar feedback. Hereafter, the widely accepted definition of young massive clusters (YMCs) is adopted, i.e., star clusters which have a present-day photometric mass exceeding $M_{\text {phot }} \gtrsim 10^{4} M_{\odot}$ and are younger than $t \lesssim 100 \mathrm{Myr}$ by stellar age. The youngest subset of them, of $\lesssim 4$ Myr age, are commonly referred to as 'starburst' clusters. These limits are not robust but generally serve as representative values.

\section{Secular expansion of star clusters}

A possible way in which a star cluster can appear within a molecular-gas filament is through a localized but intense star formation at a privileged location in the filament, say, at a junction of multiple filaments. Such a region is prone to reach a high SFE due to lateral contraction of the filaments and ample gas supply through them. Any such stellar cluster (or sub-cluster) should adapt to the compact cross-section of the filament (see Sec. 1). While formation of proto-stars outside the filaments, i.e., in rarer gas, cannot be ruled out, both observations (e.g., Schneider et al. 2012; André et al. 2014) and hydrodynamic calculations (e.g., Bate \& Bonnell 2004; Girichidis et al. 2011) suggest that the majority of the proto-stars must form within the compact dimensions of the dense gas filaments. Now, a cluster with $r_{\text {eff }} \lesssim 0.3 \mathrm{pc}$ is way more compact than the presently observed YMCs (Portegies Zwart et al. 2010), c.f. Fig. 1. The key question 
here is whether such a compact star cluster can expand by its own, through its secular evolution, to attain the presently observed sizes.

To reach the observed sizes, such a compact cluster must expand by a factor of $\gtrsim 10$. For a young, massive cluster, several effects contribute to its secular expansion. In the earliest stage (until $t \approx 4.5 \mathrm{Myr}$ ), mass loss due to stellar evolution is the primary driver of the expansion. The most massive stars remain segregated (either primordially or dynamically) at the cluster's center, causing centrally localized mass depletion due to the massive (O-type) stars' strong winds. When these stars undergo supernove, the central mass loss becomes even more severe, causing a higher rate of cluster expansion. This stellar mass loss dominated expansion continues for $\approx 50 \mathrm{Myr}$. After the stellar mass loss phase, the cluster expansion continues to be driven by dynamical heating due to the centrally segregated black holes (hereafter BHs) (Mackey et al. 2008; Banerjee et al. 2010; Morscher et al. 2013). Finally, young star clusters are observed to contain a substantial fraction ( $\gtrsim 70 \%)$ of tight primordial binaries of massive main-sequence stars (Sana \& Evans 2011). These primordial binaries also inject energy to the cluster and contribute to its expansion through binary-single and binary-binary encounters and the associated binary heating and ejections of massive stars (Heggie \& Hut 2003).

To compute the secular evolution of model massive clusters in a realistic manner, the direct N-body code NBODY7 (Aarseth 2012) is used. In addition to computing the individual stars' orbits using the highly accurate fourth-order Hermite scheme and dealing with the diverging gravitational forces, e.g., during close encounters and in hard binaries through regularizations, NBODY7 includes stellar and binary evolution recipes. The details of the computed models are given in Table. 1. Some of the models contain a primordial binary population, to assess the role of the latter in expanding a young cluster. In these models, a $f_{b}(0)=100 \%$ primordial binary fraction that follow the "birth orbital period distribution" (Kroupa 1995) is used. For massive stars of $m>5 M_{\odot}$, a much tighter period distribution, given by a (bi-modal) Öpik law (uniform distribution in $\log _{10} P$ ) for $0.3<\log _{10}(P /$ day $)<3.5$ (Sana \& Evans 2011), is used.

The solid curves in Fig. 1 shows the computed evolution of effective radius, $r_{\mathrm{eff}}(t)$, for the models in Table 1. Here, the instantaneous $r_{\text {eff }}(t)$ is obtained by taking the mean of the projected half-mass radii (50\% Lagrange radius integrated over a plane) over three mutually perpendicular planes passing through the cluster's density center. As seen, starting from sizes similar to that of the filamentary substructures in molecular clouds (see Sec. 1), it is practically impossible to attain the observed sizes of YMCs and associations in 100 Myr. Notably, realistic conditions are used in these models including stellar mass loss, retention of $\approx 50 \%$ of the BHs and NSs formed via supernovae and a realistic population of tight massive primordial binaries. A few test models also start with more extended size, $r_{h}(0) \approx 1.0 \mathrm{pc}$, which can nearly reach the sizes of the most compact observed YMCs, but still are much more compact than most YMCs. As elaborated in Banerjee \& Kroupa (2015c), theoretical uncertainties in stellar and binary evolution and core-collapse supernova, would modify the $r_{\text {eff }}(t)$ curve to some extent, but are unlikely to alter the above overall inference. This necessitates additional expansion mechanisms for a newly assembled compact cluster to reach the present-day observed sizes.

\section{Non-secular evolution of star clusters: primordial gas expulsion}

The calculations in Sec. 2 do not consider any primordial gas present initially in the proto-cluster, i.e., the cluster is taken to be formed with $100 \%$ local SFE. A high local SFE has been claimed by several hydrodynamics-based studies (e.g., Bate \& Bonnell 2004; Dale et al. 2015). Clearly, as shown in Sec. 2, if a cluster is hatched along a 


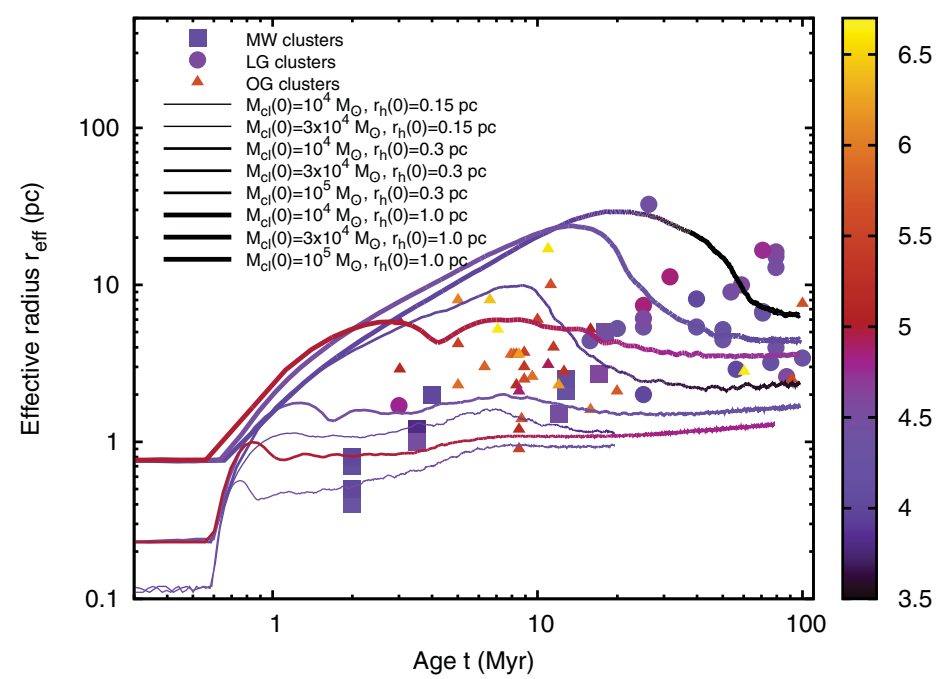

Figure 2. The data points and their colour-code are identical to Fig. 1. The curves show the computed evolution of the effective radius, $r_{\mathrm{eff}}(t)$, including a gas dispersal phase with star formation efficiency $\epsilon \approx 33 \%$. These curves imply that even if YMCs evolve from filament-like compact sizes, such substantial (and explosive) gas dispersal will expand them to their present observed sizes in the Milky way and in the Local Group. However, to attain the sizes of the most extended Local Group YMCs, one needs to evolve from $r_{h}(0) \gtrsim 1$ pc half-mass radii, unless such objects are low-mass cluster complexes.

molecular-gas filament or at a filament junction with effectively $100 \%$ SFE, it well falls short of observed sizes of YMCs. On the other hand, observations of molecular clouds and embedded clusters suggest that in regions of high star-formation activity, the local SFE typically varies between a few percent to $\approx 30 \%$. This is furthermore supported by high-resolution radiation magneto-hydrodynamic (RMHD) simulations of proto-star formation (Machida \& Matsumoto 2012; Bate et al. 2014). Although these computeexpensive simulations are limited to the spatial scale of a single proto-star, they suggest a maximum feedback-limited $\approx 30 \%$ SFE for proto-star formation; see Banerjee \& Kroupa (2015b) for more discussion. This implies that the SFE over a gas clump forming a population of proto-stars is also $\lesssim 30 \%$. Hence, it would be realistic to consider a protocluster (a pre-cluster cloud core) that is initially embedded in primordial gas, the gas being subsequently cleared from the system by the stellar feedbacks (e.g., radiation, outflows).

Fig. 2 shows the $r_{\text {eff }}$ evolutions of computed model clusters with $\approx 30 \%$ SFE which are compared to the observed $r_{\text {eff }}$ values of YMCs and associations (upper and lower panels respectively). Here, the 'gas' is simply a time-varying background potential mimicking the expulsion of ionized Hydrogen with sound speed, as implemented in previous studies (e.g., Kroupa et al. 2001; Banerjee \& Kroupa 2015a). With such an 'explosive' (i.e., in timescale comparable to the dynamical time of the cluster) gas expulsion of $\approx 70 \%$ by mass, the filament-like compact clusters can expand to reach the observed sizes of most of the MW YMCs (c.f. Fig. 2). However, most of the LG and OG YMCs are still a few factors larger in size than the computed models with compact initial conditions $\left(r_{h}(0) \lesssim 0.3 \mathrm{pc}\right)$. It seems likely that these extended YMCs are ensembles of closelylocated YMCs ( 10s of them), forming low-mass cluster complexes, thus being younger, low-mass versions of "faint-fuzzy" objects (Brüns et al. 2009); see Banerjee \& Kroupa (2015c) for details. 
Table 1. Initial conditions for the computed model clusters without a gas expulsion phase. The initial configurations are Plummer profiles with total mass, $M_{c l}(0)$, and half-mass radius, $r_{h}(0)$. The corresponding values of the virial velocity dispersion, $V_{*}(0)$, the crossing time, $\tau_{c r}(0)$, and the two-body relaxation time at half-mass radius, $\tau_{h}(0)$, are given. The initial clusters are in circular orbits at $R_{G} \approx 8 \mathrm{kpc}$ Galactocentric distance.

\begin{tabular}{lcccccc}
\hline \hline$M_{c l}(0) / M_{\odot}$ & $r_{h}(0) / \mathrm{pc}$ & $\begin{array}{c}\text { Primordial } \\
\text { binaries }\end{array}$ & $\begin{array}{c}\text { Mass } \\
\text { segregation }\end{array}$ & $V_{*}(0) / \mathrm{km} \mathrm{s}^{-1}$ & $\tau_{c r}(0) / \mathrm{Myr}$ & $\tau_{h}(0) / \mathrm{Myr}$ \\
\hline $10^{4}$ & 0.3 & no & no & 10.5 & 0.028 & 4.65 \\
$3 \times 10^{4}$ & 0.3 & no & no & 18.1 & 0.017 & 8.05 \\
$10^{4}$ & 1.0 & no & no & 5.8 & 0.172 & 28.30 \\
$3 \times 10^{4}$ & 1.0 & no & no & 10.0 & 0.100 & 49.02 \\
$10^{5}$ & 1.0 & no & no & 18.2 & 0.055 & 89.49 \\
$1.3 \times 10^{4}$ & 0.35 & yes & yes & 11.7 & 0.030 & 6.68 \\
$10^{4}$ & 1.0 & yes & yes & 6.0 & 0.167 & 28.30 \\
$3 \times 10^{4}$ & 1.0 & yes & yes & 10.4 & 0.096 & 49.02 \\
\hline \hline
\end{tabular}

\section{Cluster formation through hierarchical mergers}

So far, monolithic or in-situ formation of star clusters are considered. The substructured and filamentary conditions in molecular clouds (see Sec. 1) make it plausible that YMCs may also arise due to sequential mergers of less massive sub-clusters, which fall in the potential well of the molecular cloud (or clump). In particular, if the sub-clusters are 'decoupled' (e.g., as in Dale et al. 2015) from the gas, then the assembly process is independent and proceeds in a timescale longer than the free-fall time of the gas.

If a cluster has to form and evolve from a young age (a few Myr like the youngest Galactic YMCs), the sub-clusters must fall in from sufficiently close separation so that they can merge early enough. This is demonstrated in Banerjee \& Kroupa (2015a) for the case of the $\approx 1$ Myr old NGC 3603 young cluster (photometric mass $\gtrsim 10^{4} M_{\odot}$ ), for which the sub-clusters must merge from $\lesssim 2$ pc. This implies that despite star formation is often found to occur over $\gtrsim 10 \mathrm{pc}$ regions, only a part of the newly formed stellar structure (typically of pc scale) can actually comprise a YMC. There is mounting observational evidence of young stellar and/or proto-stellar sub-clusters packed within pc-scale regions (e.g., Massi et al. 2015; Román-Zúniga et al. 2015).

Moreover, as shown in Banerjee \& Kroupa (2015a), the "prompt" merger of several gas-filament-like compact sub-clusters produces a similarly compact cluster. Therefore, according to the results in Sec. 2, the observed YMC sizes would be unreachable for the newly assembled cluster via their inherent expansion alone. This also holds for the individual sub-clusters. Therefore, as demonstrated in Banerjee \& Kroupa (2015a), a post-merger explosive gas expulsion is instrumental in yielding YMCs that are like what we observe. If, on the other hand, the sub-clusters are brewed sufficiently apart that gas blow out happens in them separately prior to their merger, then the likely outcome would be a highly diffuse, massive stellar association with substructures, and it may, as a whole, be super-virial or sub-virial, e.g., Cyg-OB2 (also see Banerjee \& Kroupa 2015b).

\section{Concluding remarks}

The above study shows that if star clusters preferably appear within the overdense filaments of molecular clouds, adapting to their typical dimensions of $0.1-0.3 \mathrm{pc}$, then their self-driven expansion is generally insufficient for reaching the dimensions of observed YMCs. This is true if either the cluster forms in situ or via mergers of (closely located) sub-clusters, and holds irrespective of the newborn cluster's mass (c.f. Fig. 1). Having 
run out of the other possibilities, feedback-driven rapid expulsion of residual gas from the proto-cluster (see Sec. 3) seems to be indispensable to reach the observed sizes of YMCs. In this line of argument, an important concern is how YMCs gather $\gtrsim 10^{4} M_{\odot}$ within a few Myr, irrespective of any formation channel. Such amount of mass reservoir, over pc scale, is not immediately apparent from typical Galactic star-forming clouds. At the same time, the assembly phase of YMCs have to be short-lived ( $\lesssim 1 \mathrm{Myr})$, as discussed above, implying that it would be rare to catch an assembling starburst cluster.

The chances of finding a YMC in its assembling phase would be higher in starburst galaxies, where a much larger number of massive clusters are triggered compared to a Milky Way-like galaxy. Indeed, recent ALMA observations of the Antennae galaxy indicate still-forming, deeply-embedded stellar systems of total mass exceeding $10^{7} M_{\odot}$, which are either monolithic or distributed over a few pc (Johnson 2015; Johnson et al. 2015). In order to better understand the birth conditions of starburst clusters, improved and more exhaustive observations of starburst galaxies are necessary.

\section{References}

Aarseth, S. J. 2012, MNRAS, 422, 841

André, P., Di Francesco, J., Ward-Thompson, D., et al. 2014, in Beuther, H., Klessen, R., Dullemond, C. and Henning, Th. (Eds.) Protostars and Planets VI, University of Arizona Press, Tucson, p.27

Banerjee, S., Baumgardt, H., \& Kroupa, P. 2010, MNRAS, 402, 371

Banerjee, S. \& Kroupa, P. 2015a, MNRAS, 447, 728

Banerjee, S. \& Kroupa, P. 2015b, in S. W. Stahler eds. "The Birth of Star Clusters", Springer-Verlag, URL: https://astro.uni-bonn.de/sambaran/docs/VYMCformation_ Banerjee_KroupaV2.pdf (preprint)

Banerjee, S. \& Kroupa, P. 2015c, A\&A, submitted.

Bastian, N. \& Silva-Villa, E. 2013, MNRAS, 431, L122.

Bate M. R. \& Bonnell, I. A. 2004, in Lamers, H. J. G. L. M., Smith, L. J., Nota A. (Eds.) The Formation and Evolution of Massive Young Star Clusters, (ASP Conf. Proc. 322). San Francisco: Astronomical Society of the Pacific, 289

Bate, M. R., Tricco, T. S., \& Price, D. J. 2014, MNRAS, 437, 77

Brüns, R. C., Kroupa, P., \& Fellhauer, M. 2009, ApJ, 702, 1268

Dale, J. E., Ercolano, B. \& Bonnell, I. A. 2015, MNRAS, 451, 5506.

Girichidis, P., Federrath, C., Banerjee, R., \& Klessen, R. S. 2011, MNRAS, 413, 2741

Heggie, D. C. \& Hut, P. 2003, "The Gravitational Millon-Body Problem: A Multidisciplinary Approach to Star Cluster Dynamics". Cambridge University Press, Cambridge, UK

Johnson, K. 2015, IAU General Assembly, Meeting 29, id.2257770

Johnson, K. E., Leroy, A. K., Indebetouw, R., et al. 2015, ApJ, 806, 35

Kroupa, P. 1995, MNRAS, 277, 1507

Kroupa, P., Aarseth, S., \& Hurley, J. 2001, MNRAS, 321, 699

\&Machida, M. N. \& Matsumoto, T. 2012, MNRAS, 421, 588

Mackey, A. D., Wilkinson, M. I., Davies, M. B., \& Gilmore, G. F. 2008, MNRAS, 386, 65.

Massi, F., Giannetti, A., \& di Carlo, E. 2015, A\& $\&$, 573, id.A95.

Morscher, M., Umbreit, S.,Farr, W. M., \& Rasio, F. A. 2013, ApJ, 763, L15

Portegies Zwart, S. F., McMillan, S. L. W., \& Gieles, M. 2010, ARA\&A, 48, 431

Román-Zúniga, C. G., Ybarra, J., Megias, G., et al. 2015, ApJ, arXiv:1507.00016 (preprint)

Sana, H. \& Evans, C. J. 2011, in Neiner, C., Wade, G., Meynet, G. and Peters, G. (Eds.) Active OB Stars: Structure, Evolution, Mass Loss, and Critical Limits (IAU Symp. 272). Cambridge Univ. Press, Cambridge, 474

Schneider, N., Csengeri, T., Hennemann, M., et al. 2012, A\& A, 540, L11

Spitzer, L. Jr. 1987, "Dynamical Evolution of Globular Clusters", Princeton University Press. 